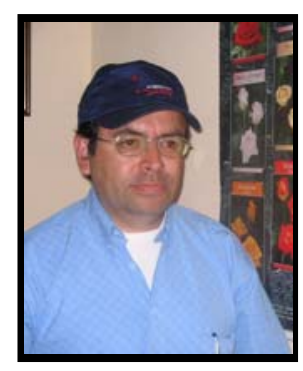

ARTigo TÉCNICO

\title{
Introdução de novas cultivares de rosas no Brasil
}

\author{
CÉSAR MAURÍCIO TORRES MARTINEZ( $\left.{ }^{1}\right)$
}

New rose variety introductions in Brazil.

In 2002 researchers, government representatives, and rose growers met in Fortaleza (Ceará) with the objective of translating rose documents from UPOV. This meeting marked the first step in protection of new rose varieties developed in Brazil, inserting that flower into the Varieties Protecting Law. Five years later many new rose varieties were registered and protected in Brazil.

There are many positive aspects that have developed during that time. A trust with Brazil occurred with breeders and international rose producers such as: Nirp International and Melland from France, Tantao from Germany and Holland, Preesman, Lex, D'Rouiters and Schuers. This occurs through participation in the Hortitec Fair every year in Holambra (São Paulo). The introduction of new Brazilian varieties has fostered an interest in improving the production, logistics and postharvest process of fresh cut roses.

Brazilian named rose varieties such as Ipanema, Copacabana, Senna, Carioca and Bahia were recognized in the last edition of Hortifair transforming these varieties into international commercialized brands, therefore bringing recognition to the Brazilian market. The Brazilian rose market is one of the biggest of the world. The efforts of the breeders union, growers and other members from commercial chains will increase the number of high quality roses. Therefore it is necessary that the governmental agencies support to Intellectual Propriety Rights of breeders and enforce laws that the illegal propagation and commercialization of protected material.

O mês de janeiro de 2002 pode ser considerado um marco histórico na rosicultura brasileira. Naquela época, reuniram-se em Fortaleza (CE), técnicos e pesquisadores do Serviço Nacional de Proteção de Cultivares, Embrapa, Secretaria de Agricultura e Pecuária do Estado do Ceará, Instituto Agropolos do Ceará e produtores de rosas estabelecidos no Estado, para traduzir documentos descritivos de rosas da UPOV para o português. Assim, foi dado o primeiro passo para facilitar a proteção de novas cultivares de rosas no Brasil, inserindo essa flor dentro da Lei de Proteção de Cultivares.

Passados quase cinco anos, mais de 48 novas variedades de rosas foram registradas e protegidas no Brasil. Vários aspectos positivos merecem destaque no decorrer de todo esse tempo:

- A confiança adquirida pelas empresas e breeders (que criam e desenvolvem novas variedades de rosas). Exemplo disso foi a participação dessas empresas, que se dedicam ao trabalho de hibridação e seleção, nos últimos dois anos na Feira Hortitec, realizada anualmente em Holambra (SP). Entre essas empresas pode-se mencionar: Nirp International e Melland, ambas francesas; Tantao, empresa alemã; e as holandesas Preesman, Lex, D’Rouiters e Schreurs;

- A implantação de estufas e campos de teste, realizada por meio de parceria entre breeders e produtores, para avaliar a adaptação de variedades nos diferentes climas do Brasil. O interesse no desenvolvimento de pólos de produção deu-se em regiões como Andradas e Barbacena (MG), Holambra e Atibaia (SP), Serra de Ibiapaba (CE) e Ipê (RS), tão diferentes em clima e solos;

- O reconhecimento por parte dos produtores profissionais com respeito à propriedade intelectual e criação de novas variedades; e

\footnotetext{
(1) Engenheiro Agrônomo, e-mail: fatorcam@hotmail.com
}

- A introdução no mercado brasileiro de mais de 40 novas variedades, oferecendo assim, alternativas de cores, formatos da flor e, inclusive, aromas, que antes não existiam no mercado nacional.

Além da introdução de novas variedades, os produtores profissionais também se preocupam em melhorar sua produção, logística e, principalmente, os processos de pós-colheita, buscando oferecer um produto de alta qualidade, e dessa forma, competindo no mercado externo com paises tradicionalmente exportadores de rosas, como Colômbia, Equador e até mesmo Kênia.

A presença de rosas brasileiras nas últimas edições da Feira Hortifair, realizada anualmente em Amsterdã (Holanda), permite a criação gradativa de uma identidade própria nacional, reforçando assim, os esforços de empresas estabelecidas no Estado do Ceará, que desde agosto de 2002 vêm exportando para os leilões holandeses.

A participação de mais de 40 produtores brasileiros em missões técnicas internacionais organizadas pela empresa de melhoramento genético de rosas NIRP International, nos dois últimos, visitando fazendas produtoras de rosas em Colômbia, Equador, Holanda e França, refletem a mudança na cultura do produtor e a necessidade de conhecer outras estratégias de manejo e especialmente de pós-colheita.

Palavras tão brasileiras como Ipanema, Copacabana, Senna, Carioca e Bahia, dão nome a variedades introduzidas nos últimos dois anos, convertendo-se assim, em marcas comerciais registradas, com maior identidade e reconhecimento à flor e, conseqüentemente, potencializando o mercado brasileiro.

Quando se faz referência aos produtores profissionais, é difícil quantificar o tamanho e o volume de produção. O compromisso se adquire com a qualidade do produto, a partir do acompanhamento de diversos fatores, 
como o manejo adequado dos recursos necessários, por exemplo, água, substrato e solo, além de outros, ainda mais importantes: o treinamento dos funcionários, seu bem-estar e de suas famílias.

Não há duvidas de que o futuro é muito promissor, uma vez que o mercado brasileiro de rosas é um dos maiores do mundo. A união de esforços entre breeders, produtores e outros membros da cadeia comercial, focados na introdução e divulgação de novas variedades, certamente incrementarão o consumo de rosas de melhor qualidade.

Para tanto, a produção profissional de rosas necessita do apoio de entidades governamentais para superar as dificuldades encontradas para se fazer respeitar os Direitos de Propriedade Intelectual dos breeders. Assim, evita-se que atos como a propagação e a comercialização ilegal de material protegido gerem problemas, como a competição desleal com os produtores que reconhecem e pagam pelo direito de explorar novas variedades de rosas. A comprovação da existência de produtores que exploram ilegalmente variedades protegidas ante o Serviço Nacional de Proteção de Cultivares, em regiões como São Sebastião do Cai (RS), Atibaia (SP) e Andradas (MG), são motivos de preocupação.

A regularização e a conscientização dos produtores que compram flores de variedades novas no mercado nacional, e que as multiplicam indiscriminadamente, são sem dúvida um dos pontos mais importantes que a indústria de rosas nacional necessita eliminar, a fim de fortalecer a confiança que as empresas, tanto as criadoras como as produtoras, depositaram na floricultura do Brasil.

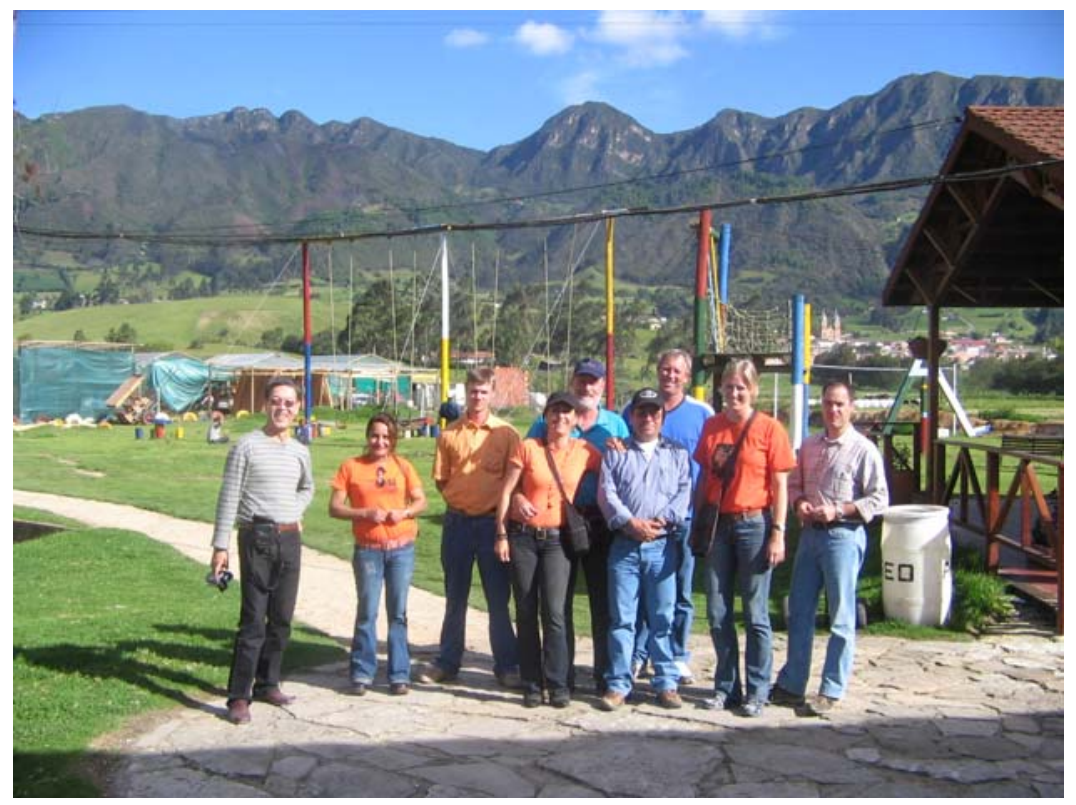

Missão técnica com produtores de rosas brasileiros na Colombia em 2005. (da esquerda para a direita) FRANCISCO SAITO, BERENIZE DA SILVA, PETER BOERSEN, ELOISAE ROBERTO KIEVITSBOSH, MAURICIOTORRES, THEODOR E PATRICIASWARTE GUSTAVO VIEIRA

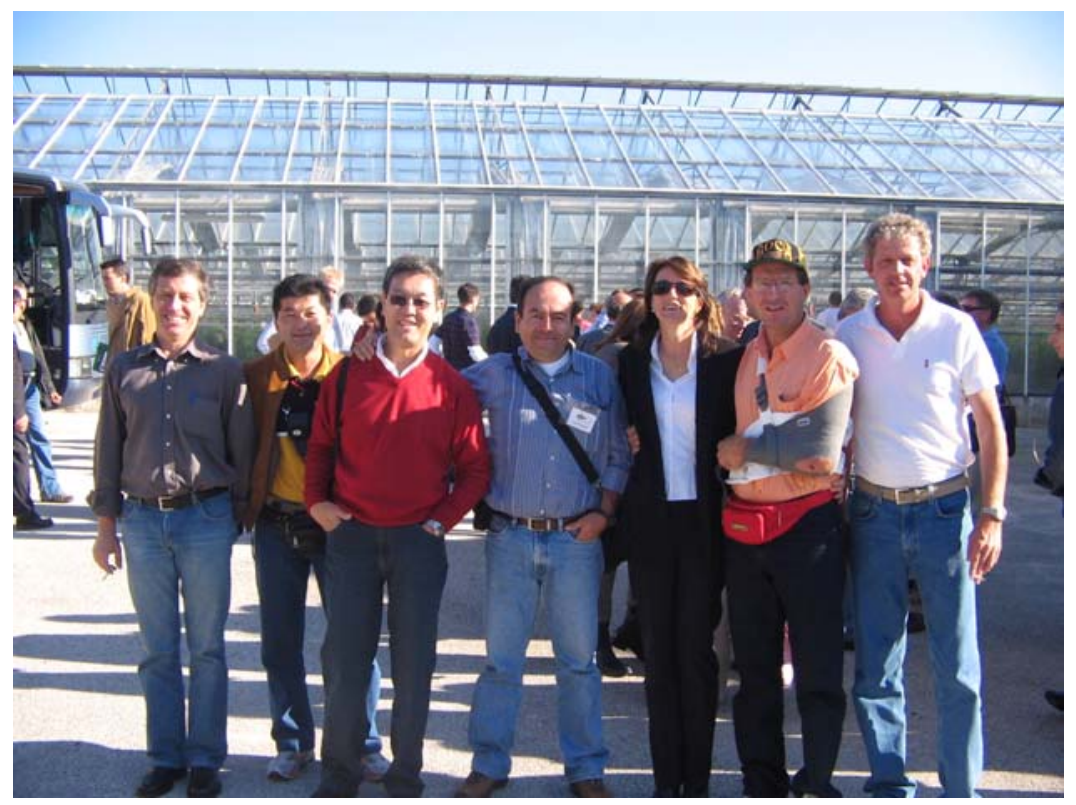

Missão Técnca na França em 2005: (da esquerda para a direita) PAULO VIADANA, CELSO OIKAWA, FRANCISCO SAITO, JACKELINE E ISIDORO DOMHOFF E ROBERTO VANROOYEN. 


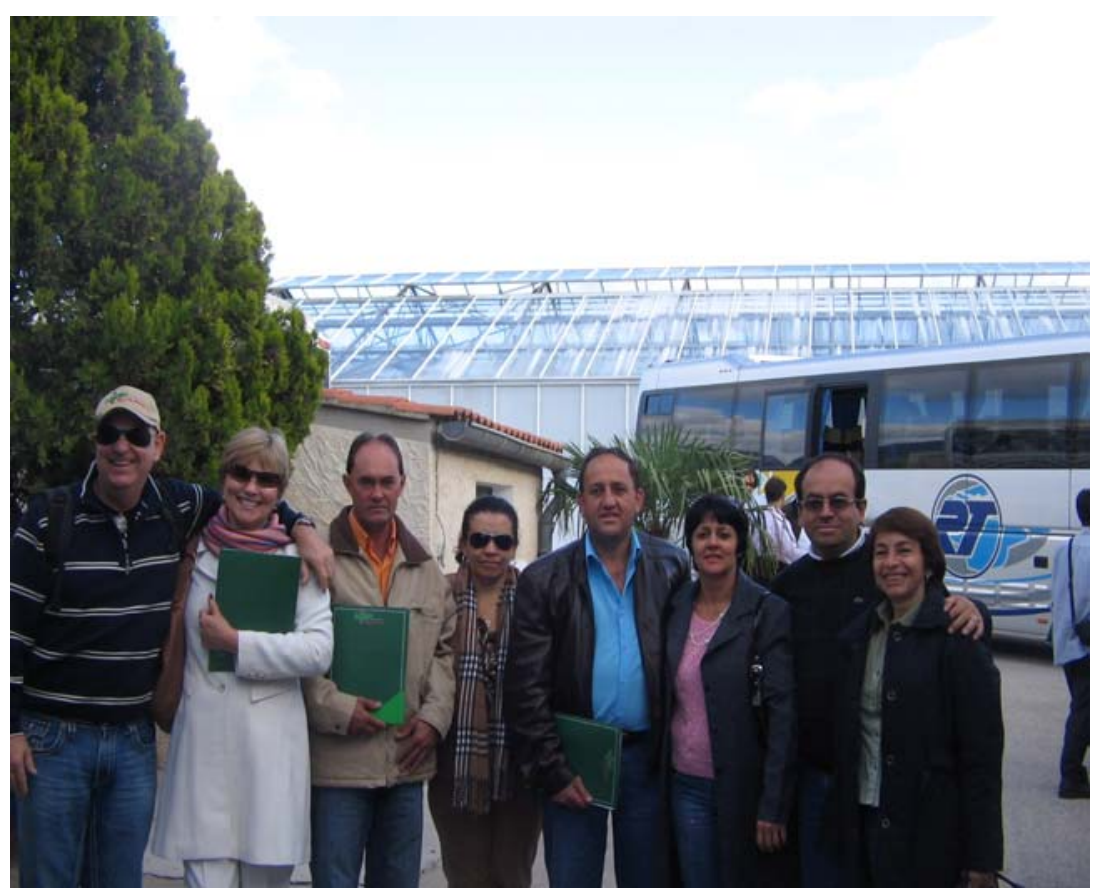

Nirp, França em 2006. (da esquerda para a direita) PAULO E SHELKA SALVACH, MAURO E MARIA FOGAÇA, MIGUEL E IVONNE ESPERANÇA E MAURICIO E ESPERANZA TORRES

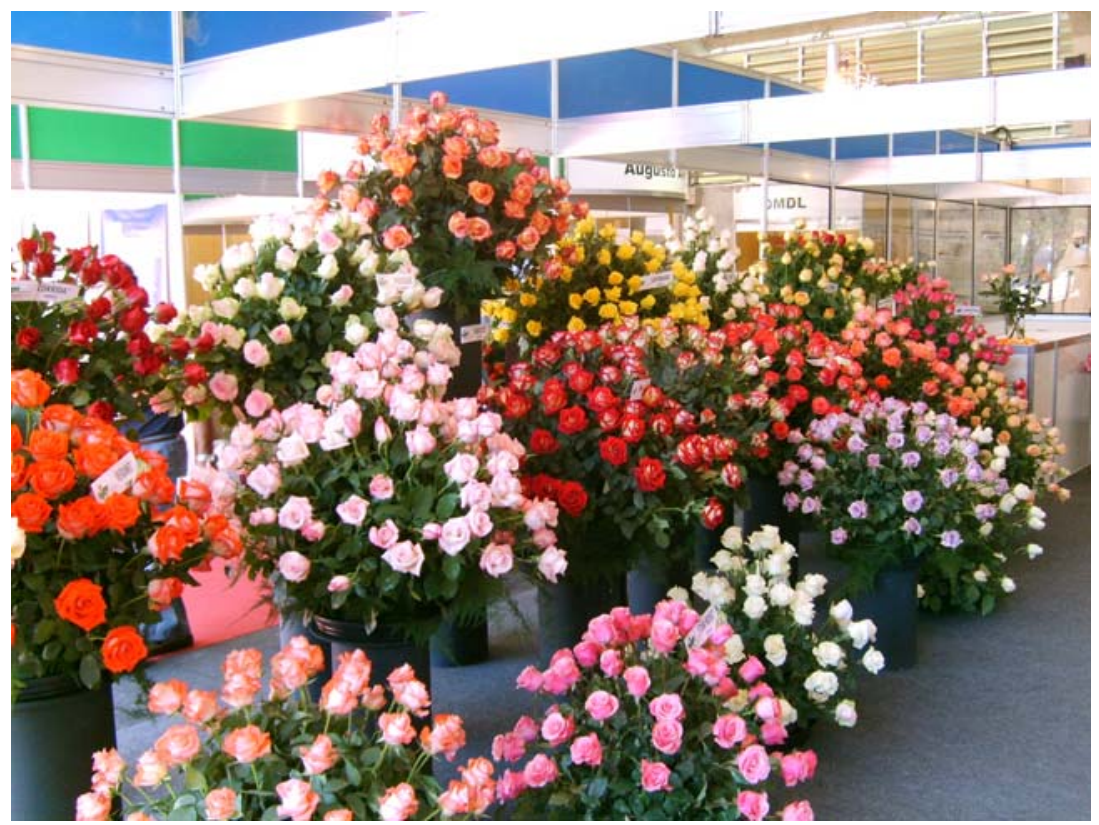

Hortitec 2006 - Diversidade das novas cultivares de rosas: rosas brasileiras de ótima qualidade 


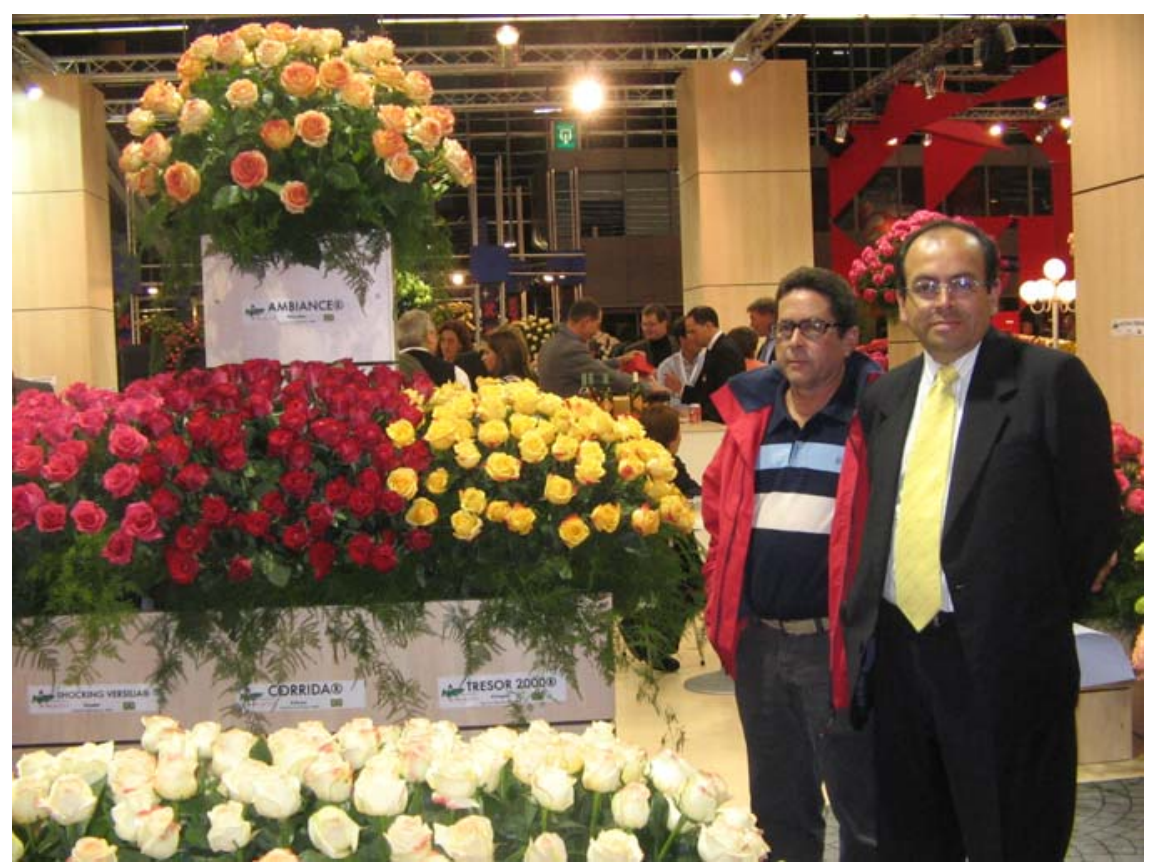

Feira Hortifair em Amsterdam 2006: SERGIO PASSOS, PRODUTOR DE ROSAS DO ESTADO DO CEARÁ E MAURICIO TORRES 